\title{
Malaria vector control in South Africa
}

\author{
B Brooke, ${ }^{1,2} \mathrm{PhD}$; L Koekemoer, ${ }^{1,2} \mathrm{PhD}$; P Kruger, ${ }^{3}$ ND Pub Hlth; J Urbach, ${ }^{4}$ MCom (Economics); E Misiani, ${ }^{5} \mathrm{PhD}$; M Coetzee, ${ }^{1,2} \mathrm{PhD}$ \\ ${ }^{I}$ Vector Control Reference Laboratory, Centre for Opportunistic, Tropical and Hospital Infections, National Institute for Communicable Diseases, \\ Johannesburg, South Africa \\ ${ }^{2}$ Wits Research Institute for Malaria, Faculty of Health Sciences, University of the Witwatersrand, Johannesburg, South Africa \\ ${ }^{3}$ Malaria Control Programme, Polokwane, Limpopo Province, South Africa \\ ${ }^{4}$ Africa Fighting Malaria, Durban, South Africa \\ ${ }^{5}$ Malaria Directorate, National Department of Health, Pretoria, South Africa
}

Corresponding author: M Coetzee (maureenc@nicd.ac.za)

\begin{abstract}
Malaria vector control is primarily insecticide based and relies on indoor residual spraying (IRS) and the distribution of long-lasting insecticide-treated bednets (LLINs). These interventions have generally proved effective where appropriately implemented. However, the increasing incidence of insecticide resistance in target vector populations can, and in several cases already has, undermined the effectiveness of IRS and LLINs. Today there are very few localities on the African continent where populations of vector mosquitoes are still susceptible to the approved classes of insecticides. If elimination is to be achieved, SA needs to invest more resources into malaria control.
\end{abstract}

S Afr Med J 2013;103(10 Suppl 2):784-788. DOI:10.7196/SAMJ.7447

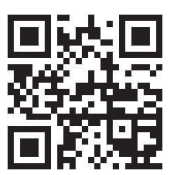

Malaria vector control is primarily insecticide based. It relies on indoor residual spraying (IRS) of houses and the distribution of long-lasting insecticide-treated bednets (LLINs). Only twelve insecticides from four different chemical classes (organochlorines, organophosphates, pyrethroids, and carbamates) are currently recommended by the World Health Organization (WHO) for IRS. ${ }^{[1]}$ The oldest classes of these insecticides are the organochlorines, of which dichlorodiphenyltrichloroethane (DDT) is the only approved insecticide, and the organophosphates, which are derived from phosphoric acid. Both have been used since the 1940s. All 12 insecticides collectively target two insect neurological sites. Currently, only pyrethroids are approved by the WHO for LLIN treatment.

These interventions have generally proved effective where appropriately implemented. ${ }^{[2]}$ However, the increasing incidence of insecticide resistance in target vector populations can, and in several cases already has, undermined the effectiveness of IRS and LLINs. ${ }^{[3-6]}$ Insecticide resistance in target vector populations, coupled with environmental and health concerns associated with insecticide use, has led to a burgeoning interest in the development of alternative forms of vector control, ${ }^{[7]}$ as well as to the implementation of integrated vector-management strategies. ${ }^{[8,9]}$

\section{Malaria mosquitoes in South Africa 1.1 Species identification}

The first essential step in a malaria vector control programme is the accurate identification of the mosquitoes involved in transmission of the disease so that scarce resources are not wasted on harmless insects. The first step in this process is the microscopic examination of the external morphology of the adults or larvae. This starts with recognition of characters that separate Anopheles mosquitoes from other culicine mosquitoes and then 'fine-tuning' the process to identify species within the genus Anopheles so as to group mosquitoes into possible vectors and non-vectors. ${ }^{[10,11]}$ However, the process does not stop there as many species are now known to belong to 'species complexes' or group of species that look identical under the microscope. These species need to be identified using modern molecular methods. One such group is the Anopheles gambiae complex. It comprises eight members of which An. gambiae, An. coluzzii and An. arabiensis are major malaria vectors, An. merus, An. melas and An. bwambae are minor/localised vectors, and An. quadriannulatus and An. amharicus are not known to transmit malaria. ${ }^{[1-1-14]}$

Similarly, An. funestus is the nominal member of a group of nine African species and the only one considered to be of any importance in malaria transmission. ${ }^{[15]}$ While other members of the group (An. parensis, An. vaneedeni and An. rivulorum) are either nonvectors or of very limited importance, the fact that they are easily confused with An. funestus using morphological characters means that accurate, molecular identification techniques developed in South Africa (SA) $)^{[1,1,17]}$ are needed for this group, just as they are for the $A n$. gambiae complex.

\subsection{Vector incrimination}

Mosquitoes are incriminated in malaria transmission based on the detection of Plasmodium sporozoites by direct dissections (Fig. 1) in the salivary glands of identified females or circumsporozoite protein (CSP) detection by enzyme-linked immunosorbent assays (ELISAs) and polymerase chain reaction (PCR). Infection rates for An. funestus in SA in the past have been recorded as high as $27 \%$ in the Letsitele Valley of Limpopo Province in the $1930 \mathrm{~s}^{[18]}$ while in the 1999/2000 epidemic the An. funestus infection rate was approximately $5 \%$ in northern KwaZulu-Natal (KZN) Province. ${ }^{[19]}$ There are no recent records of Plasmodium sporozoite-positive An. arabiensis or An. merus specimens from SA; older reports are unreliable due to lack of definitive species identification. In general, very few studies have been carried out in the past 40 years on the infectivity of South African mosquito populations ${ }^{[20,21]}$ (unpublished National Institute for Communicable Diseases (NICD) data, 2005 - 2012). By comparison, Table 1 gives sporozoite infection rates for the three species in the Maputo region of southern Mozambique, showing the clear 
impact on transmission that was achieved by the Lubombo Spatial Development Initiative (LSDI). ${ }^{[22]}$ In SA in general, An. funestus is usually associated with outbreaks and epidemics while An. arabiensis is considered largely responsible for comparatively low-level seasonal transmission in the malaria-affected regions. ${ }^{[23]}$

The study carried out by De Meillon et al.$^{[20]}$ in 1977 showed that An. vaneedeni was readily infected with Plasmodium falciparum, but this species has never been implicated in malaria transmission in nature. ${ }^{[13]}$

\subsection{Bionomics and distribution of vector species}

An. arabiensis occurs in all three of SA's malaria affected provinces. It tends to prefer dry, savannah environments and generally breeds in small, sunlit, temporary, fresh-water pools. However, breeding sites can also be found along the margins of dams in cattle hoof prints as well as in rice paddies. ${ }^{[11,13]}$ Adult females will feed on both cattle and humans and can be found resting both inside human habitations and outdoors. ${ }^{[1,13]}$ The outdoor resting components of An. arabiensis populations are largely unaffected by IRS and are almost certainly responsible for the low-level seasonal malaria transmission experienced in SA each year. ${ }^{[23]}$ The saltwater breeding

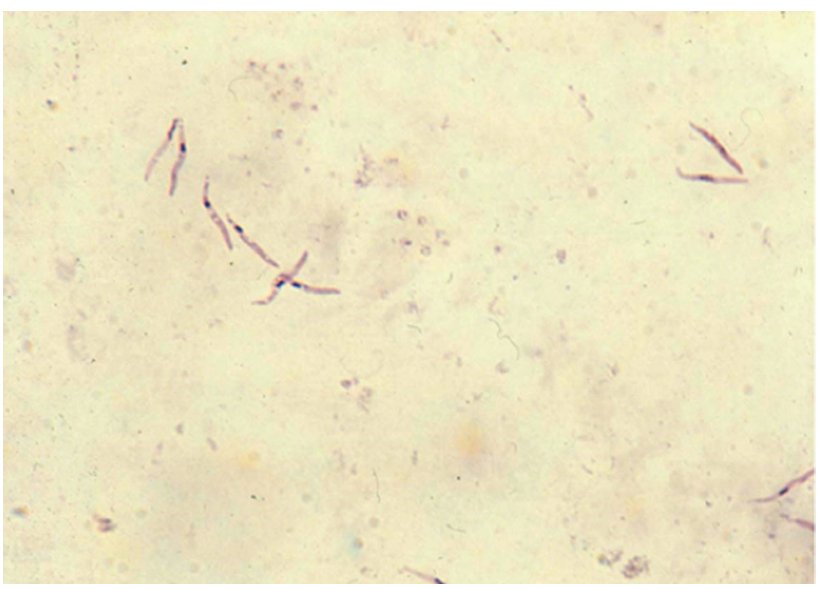

Fig. 1. Sporozoites from mosquito salivary glands. member of the An. gambiae complex, An. merus, is widespread both inland and on the coastal plains. ${ }^{[13]}$

An. funestus prefers to breed in permanent and semi-permanent fresh-water pools or slow-moving streams containing emergent vegetation. $^{[11,13]}$ Unlike An. arabiensis, this species is highly anthropophilic and endophilic making it especially amenable to control by IRS.

\section{Insecticide resistance}

Insecticide resistance in malaria vector populations is widespread. The increasing use of insecticides for malaria vector control over the past decade coupled with ongoing agricultural pesticide use has led to selection for resistance genes in a great many vector populations. ${ }^{[24]}$ Resistance to insecticides in South African malaria vector populations is a relatively recent occurrence. The first documented tests for insecticide susceptibility in local malaria vectors took place in 1978 (unpublished newsletters of the National Institute for Tropical Diseases, 1978 - 1980) with no resistance reported. No further tests were carried out until those described by Gericke et al. ${ }^{[25]}$ Samples of An. arabiensis were collected in 1996 from Thomo and Malahlapanga in Limpopo Province and Mamfene in KZN. They were assayed against DDT, deltamethrin, fenitrothion and propoxur using the standard WHO bioassay technique for testing the susceptibility of adult anopheline mosquitoes. ${ }^{[26]}$ In these tests, the Malahlapanga population was used as a control population from an insecticide-free area (Kruger National Park), against which the populations from Mamfene and Thomo were assessed, both of which are located in areas that, at that time, had experienced insecticide use either for agricultural and public health purposes (Thomo) or for public health only (Mamfene). All populations showed full susceptibility to DDT, deltamethrin and fenitrothion. The Malahlapanga and Mamfene populations also proved fully susceptible to propoxur whereas the Thomo population showed evidence of propoxur resistance. ${ }^{[25]}$

In response to the South African malaria epidemic that peaked in 2000, samples of anopheline mosquitoes were collected from the Ndumu region of northern KZN during 1999. ${ }^{[19]}$ These samples were primarily collected in window exit traps or by pyrethrum spray catches. Male anophelines, morphologically identified as members

Table 1. Sporozoite infection rates (\%) in three vector species in southern Mozambique

\begin{tabular}{lllll}
\hline Period & An. funestus & An. arabiensis & An. merus & Reference \\
\hline $1994-1996$ & 2.4 & 1.1 & - & Mendis et al., 2000 \\
1999 & 4.7 & 7.1 & 2.2 & Sharp et al., 2007 \\
2000 & 4.3 & 9.6 & 4.2 & Cuamba and Mendis, 2009 \\
2005 & 2.2 & 0.8 & 0.7 & Sharp et al., 2007
\end{tabular}

Table 2. Insecticide resistance reported in South Africa

\begin{tabular}{lllll}
\hline Insecticide & Species & Year & $\begin{array}{l}\text { 24-hour post exposure mortality } \\
\text { rate, \% (insecticide) }\end{array}$ & Reference \\
\hline DDT & An. arabiensis & 2002 & 86.5 & Hargreaves et al., 2003 $^{[30]}$ \\
& An. arabiensis & 2005 & 78 (permethrin) & Mouatcho et al., 2009 $^{[2]]}$ \\
Pyrethroid & An.funestus & 1999 & 86 (permethrin) & Hargreaves et al., 2000 $0^{[19]}$ \\
Carbamate & An. funestus & 2000 & 56 (propoxur) & Brooke et al., 2001 \\
Organophosphate & None reported & & & \\
DDT = dichlorodiphenyltrichloroethane. & & &
\end{tabular}


of the An. funestus group, that had been collected live in the window traps, were assessed for their susceptibility to permethrin in the field using a bottle bioassay. ${ }^{[27]}$ The progeny of wild-caught females, also identified as An. funestus group, were assayed under laboratory conditions for permethrin resistance using the bottle bioassay as well as the standard WHO method. ${ }^{[26]}$ Species identification of the wild mosquitoes used the new molecular method of Koekemoer et al. ${ }^{[16]}$ The insecticide bioassays revealed high levels of permethrin resistance in An. funestus but full susceptibility to DDT (Table 2). Samples of the closely related species An. rivulorum and An. parensis from the same collections gave no indication of resistance to permethrin. ${ }^{[19]}$ Subsequently, more substantial collections of An. funestus were carried out in 2000 from the Beluluane region of southern Mozambique. Resistance to the pyrethroids deltamethrin and lambda-cyhalothrin was demonstrated. Furthermore, resistance to propoxur was detected while full susceptibility to DDT was confirmed. ${ }^{[28]}$ It has since been established that southern African An. funestus are fully susceptible to the pyrrole insecticide chlorfenapyr regardless of their resistance status to pyrethroids. ${ }^{[29]}$

Samples of An. arabiensis and An. merus from the Beluluane region of southern Mozambique, collected in 2000, did not show any indication of resistance to pyrethroids (deltamethrin and lambda-cyhalothrin), DDT, carbamates (propoxur and bendiocarb) or the organophosphate fenitrothion. ${ }^{[28]}$ However, collections of An. arabiensis in 2002 from northern KZN revealed resistance to DDT, but full susceptibility to deltamethrin. ${ }^{[30]}$ Subsequent collections in Mamfene during 2005 revealed resistance to permethrin and suspected resistance to deltamethrin. ${ }^{[21]}$ At the same time, resistance to deltamethrin was also recorded in the non-vector species An. parensis from Mamfene. ${ }^{[3]]}$

\subsection{Mechanisms of resistance}

The insecticide susceptibility assays for adult mosquitoes are direct response-to-exposure tests that provide no insight on the underlying genetic mechanisms of resistance, when detected. This information can be obtained using various molecular and biochemical assays.

The elucidation of the underlying mechanisms of pyrethroid resistance in southern African An. funestus was made possible by the successful laboratory colonisation of this species using wild-caught material from southern Mozambique. ${ }^{[32]}$ It has been demonstrated that pyrethroid resistance is primarily based on the upregulated detoxifying capabilities of at least two P450 monooxygenase genes. ${ }^{[15,28,33-36]}$ The resistance phenotype is most likely inherited as a single, incompletely dominant factor and the expression of pyrethroid resistance does not affect developmental and reproductive fitness in resistant mosquitoes. ${ }^{[37,38]}$ Furthermore, the expression of pyrethroid resistance is enhanced by blood-feeding in those females carrying the resistance genotype ${ }^{[39]}$ and pyrethroid-resistant mosquitoes are likely to have thicker cuticles than their insecticide susceptible counterparts. ${ }^{[40]}$ The expression of pyrethroid resistance in southern African An. funestus generally decreases with age although this effect was mitigated by successive blood feeding and mating under laboratory conditions. ${ }^{[32,36]}$

Pyrethroid resistance in the wild population of An. arabiensis from Mamfene is also primarily based on monooxygenase detoxification. ${ }^{[21,41]}$ Microarray analysis of a DDT and pyrethroidresistant laboratory colony, which was established using wild-caught material from Mamfene, revealed over-transcription of 20 genes associated with the DDT/pyrethroid resistance phenotype. ${ }^{[42]}$ Most of these genes are monooxygenases and glutathione s-transferases. It is interesting to note that although no knockdown resistance $(k d r)$ mutations have ever been recorded in wild An. arabiensis from Mamfene, ${ }^{[2]]}$ including the most recent sample $(n=100)$ collected in 2012 (unpublished data, NICD), the laboratory colony on which these studies are based and which has been intensively selected for resistance to DDT, is now fixed for the L1014F $k d r$ mutation. Association analyses revealed that DDT and permethrin resistance in this laboratory colony is closely linked to the $\mathrm{L} 1014 \mathrm{~F}$ mutation while deltamethrin resistance is primarily based on enzyme detoxification. ${ }^{[42]}$

\subsection{Insecticide resistance management (IRM)}

The occurrence of insecticide resistance in malaria vector populations in SA necessitates the design of tailored strategies by region/province to manage resistance and thereby maintain vector control programme efficacy. As insecticide resistance has been detected in vector populations in nearly two-thirds of those countries experiencing ongoing malaria transmission, a Global Plan for Insecticide Resistance Management (GPIRM) has been developed by the WHO. ${ }^{[43]}$ This plan lists several strategies for IRM including rotations of insecticides, mosaic insecticide spraying and combinations of interventions. These are designed to preserve the efficacy of those insecticides available for public health use either by circumventing the development of resistance in target populations or by slowing the spread of resistance where it already occurs. These strategies can easily be incorporated into those programmes, based on an integrated vector management approach.

Currently, malaria vector control in SA's three malaria-affected provinces is based on an IRS mosaic approach in which pyrethroids are used for cement-brick structures while DDT is used for traditional mud-walled structures. Carbamates are also used for IRS in some instances. Cross-border malaria control initiatives with neighbouring countries ${ }^{[22]}$ also formed part of an integrated plan that was designed to achieve malaria elimination within SA's borders by $2015 .^{[44,45]}$

\section{IRS programme logistics \\ 3.1 Programmatic organisation}

While policy development on all malaria-control interventions takes place at national level, their implementation is the responsibility of the Provincial Departments of Health. Vertically-structured provincial malaria control programmes were established in KZN, Mpumalanga and Limpopo in 1995. Each province has its own malaria control structure, headed by a provincial Malaria Control Programme Manager.

IRS activities are carried out by malaria spray operators divided into malaria spray teams. These teams have bases in their respective operating areas from where IRS services are delivered to surrounding communities. The size of spray teams varies depending on the operational area, community sizes and access routes. Malaria spray teams consist of a number of spray operators $(2-10), 1$ - 2 foremen and a team leader. Teams are transported daily to their respective work areas in the communities from the malaria unit or base.

Due to the seasonal nature of malaria in SA, with IRS only taking place during the early summer, the majority of spray operators are employed as temporary workers. Malaria spray teams are supervised by qualified environmental health practitioners with reporting lines to district and provincial malaria units. Training of spray operators, in line with WHO guidelines, takes place annually. The training focuses on the correct application of insecticides, safe handling (Fig. 2) and disposal of waste.

DDT and WHO Pesticide Evaluation Scheme (WHOPES)accredited pyrethroids (e.g. deltamethrin and alpha-cypermethrin) are currently used for spraying operations. Dwellings are sprayed 


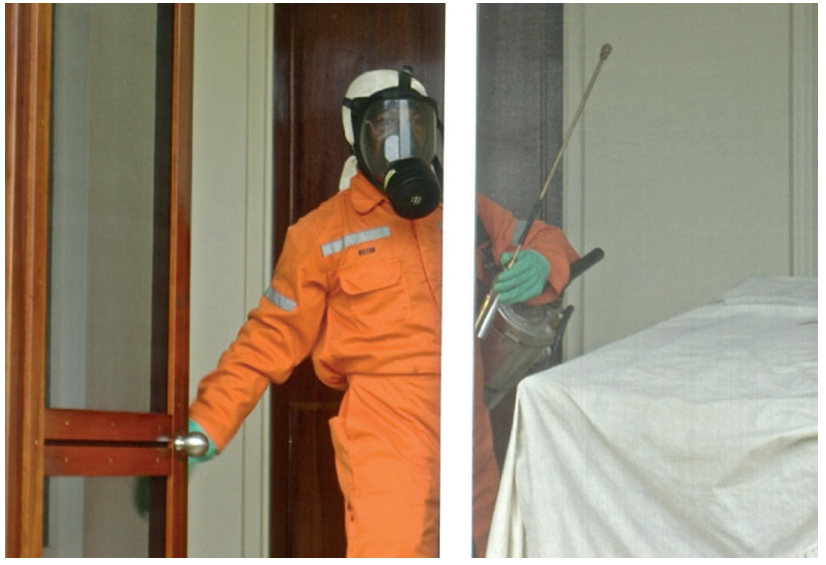

Fig. 2. Spray operators are required to wear protective clothing during indoor residual spraying operations. (Image: R. Hunt, NICD.)

before the main transmission season, commonly before the end of December. Follow-up or mop-up spraying, in areas where needed, then continues until March. At district and sub-district level there is integration and collaboration with other health programmes, e.g. Primary Health Care and Health Promotion. Approximately 1.77 million structures are covered with IRS every year in SA (National Department of Health, unpublished data).

Record-keeping systems are in place to record all IRS activities. These consist of 'hutcards' that are completed at each sprayed dwelling. Pertinent information including date, insecticide used and spray operator's details are recorded on each hutcard. This card remains with the householder for future monitoring and record purposes. The daily performance of spray operators is also recorded, with spray data entered into electronic information systems. The IRS programmes are fully funded out of each respective provincial Health Department's budget. The annual budget for malaria control at provincial level currently ranges from R35 million to R88 million depending on province and vector control accounts for about $70 \%$ of the provincial malaria budgets in any given year.

Although the IRS programmes have been highly successful in reducing malaria transmission to low levels, some operational challenges are experienced. Some of these are:

- Growth of communities in endemic areas exceeds the capacity of the malaria spray teams.

- Dwellings have become more sophisticated with extensive furnishings that inadvertently provide more vector mosquito resting sites. High IRS coverage is difficult to achieve in such dwellings. The deposits left on walls by DDT are also not always accepted by communities.

Opportunities to improve IRS through better stratification of spraying activities are currently being explored. These include the use of geographical information systems to monitor and record spray performance at household level, as well as linking IRS information to malaria case notifications within communities.

\section{Use of DDT in the malaria control programme}

The introduction of the highly effective public health insecticide, DDT, shortly after the end of World War II, allowed the malaria control programme to greatly expand its activities and dramatically reduce the burden of disease. Given that much of the cost of a malaria control spraying programme is for labour and logistics, the fact that
DDT remains effective for the whole transmission season meant that households could be protected with just one spray round per year. As a result of the expansion of the spray programme, malaria, which historically occurred as far south as Port St Johns and as far inland as Pretoria, was pushed back to the north-eastern Lowveld areas bordering Mozambique and Zimbabwe. ${ }^{[46]}$

Since the 1960s, DDT has been receiving progressively more attention from various lobby groups with respect to its harmful effects on humans and the environment, often based on suspect data. In 2000, at the height of SA's malaria epidemic, the Stockholm Convention on Persistent Organic Pollutants (POPs) held its fifth and final negotiating committee meeting in Johannesburg. The South African government, along with the assistance of international scientists and an independent advocacy group, managed to secure an exemption for those countries wishing to use DDT for malaria vector control purposes. Currently, the SC acknowledges that there is an ongoing need for DDT and allows its use for vector control according to strict guidelines drawn up by the WHO ${ }^{[47,48]}$ Countries that wish to use DDT are required to register with the Convention Secretariat and report back on a regular basis as to quantities of DDT used and what research has been undertaken to investigate equally effective, safe and affordable alternatives. To this end, some countries are being supported by the Global Environment Facility to carry out integrated vector management with the aim of reducing reliance on DDT. In the meantime, DDT remains an important insecticide in the arsenal of public health insecticides for malaria vector control.

\section{The role of entomologists in malaria vector control}

Entomological surveillance is a fundamentally important activity in a malaria vector control programme. Without skilled entomological support, analysis and decision making for IRS, particularly in terms of IRM, is not possible. Therefore, there is a need for the national and provincial malaria control programs to develop capacity within this field.

Malaria entomologists provide guidance and support to malaria vector control programmes. They should participate in the planning, implementation, monitoring and evaluation of the programme. The skills required for adequate entomological surveillance include mosquito sampling in the field, species identification using morphological and molecular methods, vector incrimination using immunological methods, and insecticide susceptibility testing using standard biological (bioassay) techniques. Follow-up operational research activities and specialised diagnostics involve more detailed and sophisticated techniques that are usually undertaken by a specialist laboratory.

The WHO has recently recommended that routine insecticide susceptibility monitoring and evaluation should be supervised by a qualified entomologist with at least an MSc degree in entomology ${ }^{[43]}$ There is currently a shortage of trained entomologists on the African continent, including SA. It is important to note that this role cannot be adequately performed by field technologists or technicians as their skills lie in a different area and are not sufficiently specialised to include entomological surveillance.

\section{Challenges for malaria vector control}

\subsection{Increasing spread of resistance}

This is an increasing problem in all African malarious countries. Today there are very few localities on the continent where populations of vector mosquitoes are still susceptible to the approved classes of 
insecticides. Pyrethroid resistance has exploded in concert with the scale-up of pyrethroid-treated bednets, causing concern for those countries that depend on LLINs for vector control. In SA, pyrethroid resistance in the Mozambican population of An. funestus remains a threat, particularly in the face of changes in housing construction in rural areas that is leading to less traditional-style housing. There are therefore fewer structures sprayed with DDT, opening the way for An. funestus to return to SA once again.

\subsection{Lack of new chemicals}

While this is being addressed by initiatives such as the Innovative Vector Control Consortium, the process is slow and expensive and it is likely that no new chemicals will be available for at least 5 years.

\subsection{Diminishing resources}

As other diseases are perceived to be more important for the health of South Africans, so resources are redirected away from malaria control. This has serious potential for us to see history repeat itself, with epidemics occurring on a scale last seen 13 years ago. If elimination is to be achieved, SA needs to invest more resources into malaria control, not less.

\section{References}

1. World Health Organization. Global Insecticide Use for Vector-Borne Disease Control. 5th ed. Geneva: WHO, 2011. http://www.who.int/iris/handle/10665/44670 (accessed 11 August 2013).

2. Pluess B, Tanser FC, Lengeler C, Sharp BL. Indoor residual spraying for preventing malaria. Cochrane Database Syst Rev 2010(4):CD006657. [http://dx.doi.org/10.1002/14651858.CD006657.pub2]

3. Govere JM, Durrheim DM, Kunene S. Malaria trends in South Africa and Swaziland and the introduction of synthetic pyrethroids to replace DDT for malaria control. S Afr J Sci 2002;98(12):19-21.

4. Coetzee M. Malaria and Dengue Vector Biology and Control in Southern and Eastern Africa. In: Knols BGJ, Louis C, eds. Bridging Laboratory and Field Research for Genetic Control of Disease Vectors. Wageningen: UR Frontis Series No. 11, 2005:101-109. [http://dx.doi.org/10.1007/1-4020-3799-6 9]

5. N'Guessan R, Corbel V, Akogbéto M, Rowland M. Reduced efficacy of insecticide-treated nets and indoor residual spraying for malaria control in pyrethroid resistance area, Benin. Emerg Infect Dis 2007;13(2):199-206. [http://dx.doi.org/10.3201/eid1302.060631]

6. Ranson H, Nguessan R, Lines J, Moiroux N, Nkuni Z, Corbel V. Pyrethroid resistance in African anopheline mosquitoes: What are the implications for malaria control? Trends Parasitol 2011;27(2):9198. [http://dx.doi.org/10.1016/j.pt.2010.08.004]

7. Takken W, Knols BG. Malaria vector control: Current and future strategies. Trends Parasitol 2009;25(3):101-104. [http://dx.doi.org/10.1016/j.pt.2008.12.002]

8. World Health Organization. Global Strategic Framework for Integrated Vector Management. Geneva: WHO, 2004. http://www.who.int/iris/handle/10665/68624 (accessed 11 August 2013).

9. Chanda E, Masaninga F, Coleman M, et al. Integrated vector management: The Zambian experience. Malar J 2008;7:164. [http://dx.doi.org/10.1186/1475-2875-7-164]

10. Gillies MT, De Meillon B. The Anophelinae of Africa South of the Sahara. 2nd ed. Johannesburg: South African Institute for Medical Research, 1968.

11. Gillies MT, Coetzee M. A supplement to the Anophelinae of Africa South of the Sahara. Johannesburg: South African Institute for Medical Research, 1987.

12. Hunt RH, Coetzee M, Fettene M. The Anopheles gambiae complex: A new species from Ethiopia. Trans R Soc Trop Med Hyg 1998;92(2):231-235. [http://dx.doi.org/10.1016/S0035-9203(98)90761-1]

13. Sinka ME, Bangs MJ, Manguin S, et al. The dominant Anopheles vectors of human malaria in Africa, Europe and the Middle East: Occurrence data, distribution maps and bionomic précis. Parasit Vectors Europe and the Middle East: Occurrence data, distributio

14. Coetzee M, Hunt RH, Wilkerson R, et al. Anopheles coluzzii and Anopheles amharicus, new member of the Anopheles gambiae complex. Zootaxa 2013;3619(3):246-274. [http://dx.doi.org/10.11646/ zootaxa.3619.3.2

15. Coetzee M, Koekemoer L. Molecular systematics and insecticide resistance in the major African malaria vector Anopheles funestus. Annu Rev Entomol 2013;58:393-412. [http://dx.doi.org/ 10.1146/ annurev-ento-120811-153628

16. Koekemoer LL, Lochouarn L, Hunt RH, Coetzee M. Single-strand conformation polymorphism analysis for identification of four members of the Anopheles funestus (Diptera: Culicidae) group. Med Entomol 1999;36(2):125-130.

17. Koekemoer LL, Kamau L, Hunt RH, Coetzee M. A cocktail polymerase chain reaction (PCR) assay to identify members of the Anopheles funestus (Diptera: Culicidae) group. Am J Trop Med Hyg 2002;66(6):804-811.

18. Swellengrebel NH, Annecke S, De Meillon, B. Malaria investigations in some parts of the Transvaal and Zululand. Johannesburg: South African Institute for Medical Research, 1931:4(27):245-274

19. Hargreaves K, Koekemoer LL, Brooke BD, Hunt RH, Mthembu J, Coetzee M. Anopheles funestus Hargreaves K, Koekemoer LL, Brooke BD, Hunt RH, Mthembu J, Coetzee M. Anopheles funestus
resistant to pyrethroid insecticides in South Africa. Med Vet Entomol 2000;14(2):181-189. [http:// dx.doi.org/10.1046/j.1365-2915.2000.00234.x]
20. de Meillon B, Van Eeden G, Coetzee L, et al. Observations on a species of the Anopheles funestus subgroup, a suspected exophilic vector of malaria parasites in northeastern Transvaal, South Africa. Mosq News 1977;37(4):657-661.

21. Mouatcho JC, Munhenga G, Hargreaves K, Brooke BD, Coetzee M, Koekemoer LL. Pyrethroid resistance in a major African malaria vector Anopheles arabiensis from Mamfene, northern KwaZuluNatal, South Africa. S Afr J Sci 2009;105:127-131.

22. Sharp BL, Kleinschmidt I, Streat E, et al. Seven years of regional malaria control collaboration Mozambique, South Africa, and Swaziland. Am J Trop Med Hyg 2007;76(1):42-47.

23. Coetzee M. Hunt RH. Malaria at its southern-most fringe in Africa. Res Rev Parasitol 1998:58(3/4):175-179.

24. Insecticide Resistance Action Committee (IRAC). 2010. Prevention and Management of Insecticide Resistance In Vectors of Public Health Importance, 2nd ed. http://www.irac-online.org/documents/ irm-mini-vector-booklet-slides/?ext=pdf (accessed 27 August 2013).

25. Gericke A, Govere, JM, Durrheim DN. Insecticide susceptibility in the South African malaria mosquito Anopheles arabiensis. S Afr J Sci 2002;98(3/4):205-208.

26. World Health Organization. Test Procedures for Insecticide Resistance Monitoring in Malaria Vectors, Bio-Efficacy and Persistence of Insecticides on Treated Surfaces. Geneva: WHO, 1998. http://www. who.int/iris/handle/10665/64879 (accessed 11 August 2013).

27. Brogdon WG, McAllister JC. Simplification of adult mosquito bioassays through use of time-mortality determinations in glass bottles. J Am Mosq Control Assoc 1998;14(2):159-167.

28. Brooke BD, Kloke G, Hunt RH, et al. Bioassay and biochemical analyses of insecticide resistance in southern African An. funestus (Diptera: Culicidae). Bull Entomol Res 2001;91(4):265-272. [http:// dx.doi.org/10.1079/BER2001108]

29. Oliver SV, Kaiser ML, Wood OR, Coetzee M, Rowland M, Brooke BD. Evaluation of the pyrrole insecticide chlorfenapyr against insecticide susceptible and pyrethroid resistant Anopheles funestus (Diptera: Culicidae). Trop Med Int Health 2010;15(1):127-131. [http://dx.doi.org/10.1111/j.13653156.2009.02416.x

30. Hargreaves K, Hunt RH, Brooke BD, et al. Anopheles arabiensis and An. quadriannulatus resistance to DDT in South Africa. Med Vet Entomol 2003;17(4):417-422. [http://dx.doi.org/10.1111/j.13652915.2003.00460.x]

31. Mouatcho JC, Hargreaves K, Koekemoer LL, et al. Indoor collections of the Anopheles funestus group (Diptera: Culicidae) in sprayed houses in northern KwaZulu-Natal, South Africa. Malar J 2007;6:30, [http://dx.doi.org/10.1186/1475-2875-6-30]

32. Hunt RH, Brooke BD, Pillay C, Koekemoer LL, Coetzee M. Laboratory selection for and characteristics of pyrethroid resistance in the malaria vector Anopheles funestus. Med Vet Entomol 2005;19(3):271275. [http://dx.doi.org/10.1111/j.1365-2915.2005.00574.x]

33. Amenya DA, Koekemoer LL, Vaughan A, et al. Isolation and sequence analysis of P450 genes from a pyrethroid resistant colony of the major malaria vector Anopheles funestus. DNA Seq 2005;16(6):437pyrethroid resistant colony of the major malaria vecto

34. Amenya DA, Naguran R, Lo T-C M, et al. Overexpression of a cytochrome P450 (CYP6P9) in a major African malaria vector, Anopheles funestus, resistant to pyrethroids. Insect Mol Biol 2008;17(1):19-25. [http://dx.doi.org/10.1111/j.1365-2583.2008.00776.x]

35. Wondji CS, Irving H, Morgan J, et al. Two duplicated P450 genes are associated with pyrethroid resistance in Anopheles funestus, a major malaria vector. Genome Res 2009; 19(3): 452-459. [http:// dx.doi.org/10.1101/gr.087916.108]

36. Christian R, Matambo TS, Spillings BL, Brooke BD, Coetzee M, Koekemoer LL. Age related pyrethroid resistance is not a function of P450 gene expression in the major African malaria vector, Anopheles funestus (Diptera: Culicidae). Genet Mol Res 2011;10(4):3220-3229. [http://dx.doi.org/10.4238/2011. December.21.4]

37. Okoye PN, Brooke BD, Hunt RH, Coetzee M. Relative developmental and reproductive fitness associated with pyrethroid resistance in the major southern African malaria vector Anopheles funestus. Bull Entomol Res 2007;97(6):599-605. [http://dx.doi.org/10.1017/S0007485307005317]

38. Okoye PN, Brooke BD, Koekemoer LL, Hunt RH, Coetzee M. Inheritance of pyrethroid resistance in the major southern African malaria vector Anopheles funestus. Ann Trop Med Parasitol 2008:102(3):275-281. [http://dx.doi.org/10.1179/136485908X278748]

39. Spillings BL, Coetzee M, Koekemoer LL, Brooke BD. The effect of a single blood meal on the phenotypic expression of insecticide resistance in the major malaria vector Anopheles funestus Giles. Malar J 2008;7:226. [http://dx.doi.org/10.1186/1475-2875-7-226]

40. Wood OR, Hanrahan S, Coetzee M, Koekemoer LL, Brooke BD. Cuticle thickening associated with pyrethroid resistance in the malaria vector Anopheles funestus. Parasit Vectors 2012;3:36. [http:// dx.doi.org/10.1186/1756-3305-3-67]

41. Munhenga G, Koekemoer LL. Differential expression of P450 genes in Anopheles arabiensis following permethrin selection. Afr J Biotechnol 2011;10(59):12711-12716. [http://dx.doi.org/10.5897/ AJB11.363]

42. Nardini L, Christian RN, Coetzer N, Ranson H, Coetzee M, Koekemoer LL. Detoxification enzymes associated with insecticide resistance in laboratory strains of Anopheles arabiensis of different geographic origin. Parasit Vectors 2012;5:113. [http://dx.doi.org/10.1186/1756-3305-5-113]

43. World Health Organization. Global Plan for Insecticide Resistance Management in Malaria Vectors (GPIRM). Geneva: WHO, 2012. http://www.who.int/iris/handle/10665/44846 (accessed 11 August 2013).

44. Moonasar D, Nuthulaganti T, Kruger PS, et al. Malaria control in South Africa 2000-2010: Beyond MDG6. Malar J 2012;11:294. [http://dx.doi.org/10.1186/1475-2875-11-294]

45. Maharaj R, Morris N, Seocharan I, et al. The feasibility of malaria elimination in South Africa. Malar I 2012;11:423. [http://dx.doi.org/10.1186/1475-2875-11-423]

46. Mabaso MLH, Sharp B, Lengeler C. Historical Review of Malaria Control in southern Africa with emphasis on the use of indoor residual spraying. Trop Med Int Health 2004;9(8):846-856. [http:// dx.doi.org/10.1111/j.1365-3156.2004.01263.x

47. United Nations Environment Programme. Stockholm Convention on Persistent Organic Pollutants (POPs). UNEP/Chemicals/2001/3.50p. 2001. http://chm.pops.int/Convention/Publications/Other/ tabid/3072/Default.aspx (accessed 11 August 2013).

48. World Health Organization, Global Malaria Programme. The Use of DDT in Malaria Vector Control: World Health Organization Position Statement, 2011. http://whqlibdoc.who.int/hq/2011/WHO_ HTM_GMP_2011_eng.pdf (accessed 11 August 2013).

49. Mendis C, Jacobsen JL, Gamage-Mendis A, et al. Anopheles arabiensis and An. funestus are equally important vectors of malaria in Matola coastal suburb of Maputo, southern Mozambique. Med Vet Entomol 2000;14(2):171-180. [http://dx.doi.org/10.1046/j.1365-2915.2000.00228.x]

50. Cuamba N, Mendis C. The role of Anopheles merus in malaria transmission in an area of southern Mozambique. J Vector Borne Dis 2009;46(2):157-159. 